$\$$ Research Square

\title{
Nivolumab augments antitumor activity of invariant natural killer T cells
}

\section{Mami Negawa}

Chiba Daigaku Daigakuin Igaku Kenkyuin Igakubu

\section{Fumie Ihara}

Chiba Daigaku Daigakuin Igaku Kenkyuin Igakubu

Shinichiro Motohashi ( $\nabla$ motohashi@faculty.chiba-u.jp )

https://orcid.org/0000-0002-9332-3129

\section{Research note}

Keywords: NKT cell, nivolumab, combination therapy, NKT cell-based immunotherapy, antitumor immunity, PD-1

Posted Date: January 7th, 2020

DOI: https://doi.org/10.21203/rs.2.20206/v1

License: (9) This work is licensed under a Creative Commons Attribution 4.0 International License. Read Full License 


\section{Abstract}

Objective: Programmed death-1 (PD-1) negatively regulates T cell functions. Nivolumab is a clinically approved anti-PD-1 antibody that inhibits PD-1 signaling, thus enhancing T cell activity. Although nivolumab has been widely used in the treatment of various cancers, it is only effective in limited patients. To develop better treatment options, combination therapies with nivolumab have been in the spotlight. Natural killer T (NKT) cell-based immunotherapy involves activation of NKT cells by injection of ligand-loaded dendritic cells, thereby inducing antitumor immunity. In this study, we examined whether nivolumab treatment enhances NKT cell activity in tumor immunity to investigate the potential use of nivolumab in NKT cell-based immunotherapy as a combination therapy to improve the current treatment options. Results: PD-1 expression in NKT cells was upregulated in response to T cell receptor stimulation. Although nivolumab treatment had no impact on NKT cell proliferation, nivolumab-treated NKT cells exhibited increased production of cytokines and Granzyme B. Furthermore, nivolumab treatment significantly enhanced the cytotoxic activity of NKT cells against K562 or A549 cells and had an adjuvant effect on natural killer cell function. Taken together, these data indicate that combination of NKT cellbased immunotherapy and nivolumab treatment could be a better treatment option for various cancer.

\section{Introduction}

Invariant natural killer T (NKT) cells are a unique subpopulation of lymphocytes characterized by expression of invariant T cell receptor (TCR) Va24 and V $\beta 11$ chains in humans. NKT cells abundantly produce various kinds of cytokines and cytotoxic factors soon after stimulation with a specific glycolipid ligand, a-galactosylceramide ( $a-G a l C e r)$, presented by CD1d on antigen-presenting cells (APCs) 11,2$]$. NKT cells exert strong tumoricidal effects both directly or indirectly via activation of other immune cells[3]. Based on these potent cytotoxic effects of activated NKT cells, we developed an NKT cell-based immunotherapy for patients with lung cancer or head and neck cancer $[4,5]$. In our previous clinical trials, increased interferon (IFN)-y production by peripheral blood mononuclear cells (PBMCs) and clinical benefits were observed in some patients with non-small cell lung cancer and head and neck cancers[6]. However, the clinical efficacy of the therapy was limited in other patients. Therefore, strategies are needed to overcome these limitations for better treatment.

Recently, programmed death (PD)-1/PD ligand (PD-L) signaling was reported to play a crucial role in immunosuppression by tumors[7]. PD-1 is an inhibitory receptor expressed on activated T cells, natural killer (NK) cells, and other immune cells[8-10]. The interaction of PD-1 with PD-L1/2 negatively regulates the effector phase of their cellular functions. Blockade of PD-1/PD-L signaling can augment antitumor responses and facilitate tumor rejection[11, 12]. It was previously shown that NKT cells express PD-1 on their surface and that blockade of PD-L1 on APCs enhances NKT cell function[13]. Nivolumab is a human IgG4 that binds to PD-1 with high affinity and inhibits PD-1/PD-L signaling, leading to recovery of conventional $\mathrm{T}$ cell function from the suppressed state[14]. The present study aimed to assess the combination of NKT cell-based immunotherapy and nivolumab treatment. 


\section{Materials And Methods}

\section{Cell lines and medium}

The human leukemia cell line K562 was kindly provided by Dr. C. H. June[15] and the non-small-cell lung cancer cell line A549 was kindly provided by Dr. K. Suzuki[16]. All cells were maintained in RPMI 1640 medium (FUJIFILM Wako Pure Chemical Corporation, Osaka, Japan) supplemented with 10\% FBS, 50 mM b-mercaptoethanol (Thermo Fisher Scientific, Waltham, MA), 10 mM Hepes (Thermo Fisher Scientific), and penicillin-streptomycin (Thermo Fisher Scientific).

\section{Flow cytometry}

Cells were stained with the following antibodies: anti-TCR Va24-FITC (clone C15) and anti-TCR Vb11-PE (clone C21) from Beckman Coulter (Brea, CA); anti-CD56-FITC (clone NCAM16) from BD Biosciences (Franklin Lakes, NJ); anti-PD-1-Pacific Blue (clone EH12.EH7) and anti-CD3-APC/Cy7 (clone HIT CD3a) from Biolegend (San Diego, CA). Data were collected by a FACSCanto II flow cytometer (BD Biosciences), and analyzed using FlowJo software (FlowJo LLC, Ashland, OR).

\section{Cell preparation and culture}

PBMCs were isolated from peripheral blood of healthy donors by density gradient centrifugation using Ficoll-Paque PLUS (GE Healthcare, Chicago, IL). For NKT cell expansion, PBMCs were cultured in RPMI 1640 medium in the presence of $200 \mathrm{ng} / \mathrm{mL}$ a-GalCer (REGiMMUNE, Tokyo, Japan) and $100 \mathrm{JRU} / \mathrm{mL}$ recombinant human IL-2 (Shionogi, Osaka, Japan) for 6 days. Nivolumab or ultra-LEAF Purified Human IgG4 recombinant antibody (Biolegend) were added to the culture on days 0,3 , and 5 . Nivolumab was provided by Ono Pharmaceutical Co. Ltd. (Osaka, Japan). After 6 days of culture, NKT cells were labeled with the anti-TCR Va24-FITC antibody, followed by positive selection using anti-FITC MicroBeads and an autoMACS Pro Separator (Miltenyi Biotec, Bergisch Gladbach, Germany). Subsequently, purified NKT cells ( $>90 \%$ purity) were rested in RPMI 1640 medium in the presence of $100 \mathrm{JRU} / \mathrm{mL}$ IL-2 overnight and used in further experiments.

\section{Cytotoxicity assay of NKT cells}

The K562 and A549 cancer cell lines were labeled with an Invitrogen CellTrace Violet Cell Proliferation Kit (CTV) (Thermo Fisher Scientific), in accordance with the manufacturer's protocol. NKT cells preincubated with $10 \mu \mathrm{g} / \mathrm{mL}$ nivolumab or $10 \mu \mathrm{g} / \mathrm{mL}$ hlgG4 isotype control for 30 min were co-cultured with the cancer cell lines at E/T ratios of 2.5:1, 5:1, and 10:1 in 96-well plates for 4-6 h. The cells were then stained with AnnexinV-FITC and PI using an Invitrogen Dead Cell Apoptosis Kit (Thermo Fisher Scientific), according to the manufacturer's protocol. $\mathrm{CTV}^{+}$AnnexinV ${ }^{+}$cells were considered apoptotic cancer cells. Cytotoxicity was calculated by the following equation: Cytotoxicity $(\%)=\mathrm{CTV}^{+}\left[\%\right.$ AnnexinV $^{+}$target cells $-\%$ Annexin $\mathrm{V}^{+}$spontaneous cells]. 
To assess the expression levels of effector molecules, NKT cells and K562 cells were co-cultured at an E/T ratio of 10:1, harvested for RNA isolation, and subjected to real-time PCR.

\section{Cytotoxicity assay of NK cells co-cultured with NKT cells}

NK cells were negatively selected from PBMCs using an NK Cell Isolation Kit (Miltenyi Biotec) and the autoMACS Pro Separator. NKT cells cultured with nivolumab or the isotype control were seeded in the upper compartment of transwells separated by a $0.4-\mu \mathrm{m}$ pore membrane with $\mathrm{K} 562$ or A549 cells at an $\mathrm{E} / \mathrm{T}$ ratio of 10:1. At $4 \mathrm{~h}$ after NKT cell seeding, NK cells were seeded in the lower compartment with CTVlabeled K562 cells at an E/T ratio of 1:1 or CTV-labeled A549 cells at an E/T ratio of 2:1. The lower compartments containing cells were harvested after $4 \mathrm{~h}$ and the cytotoxicity of NK cells was assessed as described above.

\section{RNA isolation and quantitative real-time PCR}

Total RNA was isolated using Invitrogen TRIzol Reagent (Thermo Fisher Scientific). cDNA was synthesized using Invitrogen SuperScript IV VILO Master Mix (Thermo Fisher Scientific). Real-time PCR was performed using Applied Biosystems TaqMan ${ }^{\mathrm{TM}}$ Fast Universal PCR Master Mix (Thermo Fisher Scientific) with an Applied Biosystems Step One Plus Real-time PCR system (Thermo Fisher Scientific). Primers and probes were purchased from Roche (Basel, Switzerland). Relative gene expression was calculated by the DDCt method using HPRT1 as a reference gene for normalization. The primer sequences were: Granzyme B: 5'-AGATGCAACCAATCCTGCTT-3' and 3'-CATGTCCCCCGATGATCT-5'; Perforin: 5'-CACTCACAGGCAGCCAACT-3' and 3'-GGGAGTGTGTACCACATGGA-5'; TNF-a: 5'CAGCCTCTTCTCCTTCCTGAT-3' and 3'-GCCAGAGGGCTGATTAGAGA-5'; TRAIL: 5'TCACAGTGCTCCTGCAGTCT-3' and 3'-GCCACTTTTGGAGTACTTGTCC-5'; FASL: 5'AGCTGGCAGAACTCCGAGAG-3' and 3'-GGCATGGACCTTGAGTTGGA-5'.

\section{Statistical analysis}

Statistical analysis was performed using GraphPad PRISM software version 6.0b (GraphPad Software, San Diego, CA). Data were presented as mean \pm standard deviation. Values of $P<0.05$ were considered statistically significant.

\section{Results And Discussion}

First, we sought to determine whether PD-1 expression was altered upon activation of NKT cells. For this, we stimulated NKT cells among PBMCs with a-GalCer and assessed their surface expression of PD-1. PD1 was expressed on NKT cells at a basal level prior to stimulation and dramatically increased after 3 days of stimulation (Fig. 1A, 1B). PD-1 expression was further upregulated until 5 days of stimulation and then gradually decreased, suggesting that PD-1 signaling may affect NKT cells during the relatively early phase of activation. Because nivolumab treatment enhances the proliferation and function of 
conventional T cells through blockade of PD-1 signaling[17], we also examined whether nivolumab treatment affected NKT cell expansion. After 7 days of culture, similar NKT cell percentages and numbers were observed between nivolumab-treated NKT cells and control IgG4-treated NKT cells (Fig 1C, 1D). To further determine whether nivolumab treatment altered NKT cell functions, we evaluated the production of cytokines and Granzyme B by NKT cells. NKT cells were stimulated with a-GalCer-loaded plate-bound CD1d dimers in the presence of plate-bound PD-L1 chimeric protein, to create artificial PD-1 signaling. NKT cells produced large amounts of IFN-g, IL-4, TNF-a, and Granzyme B under this stimulation condition (Fig. 1E). When NKT cells were treated with nivolumab, the production of cytokines and Granzyme B was further enhanced compared with control IgG treatment. Taken together, nivolumab blocks PD-1/PD-L1 signaling in NKT cells, thereby enhancing cytokine production but not affecting cell proliferation.

NKT cells are known to exert direct cytotoxicity against cancer cells and play an important role in antitumor immunity. Thus, we sought to determine whether nivolumab treatment enhanced the tumoricidal activity of NKT cells. For this, NKT cells treated with nivolumab or control IgG4 were cocultured with $\mathrm{K} 562$ or A549 cells and tumor cell-specific apoptosis was assessed by annexinV staining. Because NKT cells are known to target these cell lines and exert tumoricidal activity, nivolumab treatment was expected to increase the tumoricidal activity of NKT cells. As expected, NKT cells treated with nivolumab induced more apoptosis in both $\mathrm{K} 562$ and A549 cells compared with control IgG4-treated cells when co-cultured at an E/T ratio of 10:1 (Fig. 2A). NKT cells treated with nivolumab induced higher rates of apoptosis in K562 cells compared with control IgG4-treated cells at ratios of 10:1, 5:1, and 2.5:1 (Fig. 2B). We observed the same trend from three different donors, with higher apoptosis induction in K562 and A549 cells when co-cultured with nivolumab-treated NKT cells compared with control IgG4-treated cells (Fig. 2C). To dissect the mechanisms by which nivolumab-treated NKT cells induced higher rates of apoptosis in tumor cells, the expression of cytotoxic molecules was assessed after co-culture of NKT cells and K562 cells at a E/T ratio of 10:1. The expression levels of Granzyme B, Perforin, TNF-a, TRAIL, and FASL were higher in nivolumab-treated NKT cells compared with control IgG4-treated cells (Fig. 2D), indicating that nivolumab treatment elevated the expression of cytotoxic molecules in NKT cells, thus augmenting the tumoricidal activity of NKT cells.

Another important role of NKT cells in antitumor immunity is their orchestration of other immune cells such as NK cells and cytotoxic CD8 ${ }^{+} \mathrm{T}$ cells through the production of cytokines. IFN-g is a one of the major cytokines that increases the cytotoxic functions of these other immune cells. Because we observed elevated IFN-g production by NKT cells with nivolumab treatment, we further investigated whether nivolumab-treated NKT cells induced stronger tumoricidal activity of NK cells as an adjuvant effect. For this, we used a transwell culture system and cultured NK cells and NKT cells in separate compartments in the presence of K562 or A549 cells. There was no increase in the cytotoxicity of NK cells against K562 or A549 cells in the presence of control IgG4-treated NKT cells. In contrast, the cytotoxicity of NK cells was significantly enhanced by nivolumab-treated NKT cells (Fig. 3A, 3B). These data indicate that nivolumabtreated NKT cells have an enhanced adjuvant effect on NK cells. 
Herein, we have shown that nivolumab treatment enhanced overall NKT cell activity. Although nivolumab had no effect on NKT cell expansion, it enhanced cytokine production and had an increased adjuvant effect on NK cells. Because blockade of PD-1/PD-L1 signaling in conventional T cells enhances both their proliferation and effector functions, different mechanisms may control the proliferation of NKT cells and conventional T cells. Nivolumab treatment also elevated the tumoricidal activity of NKT cells. These data suggest that combination therapy of nivolumab treatment and NKT cell-based immunotherapy may be a powerful tool for treatment of cancer patients. We previously reported that blockade of PD-L1 expressed on APCs augmented NKT cell functions[13]. We confirmed that blockade of PD-1 signaling by nivolumab treatment had the same effect. Because nivolumab is a clinically approved drug, its use in NKT cell-based immunotherapy would be an advantage for application in the clinical field. The present data suggest that combination therapy of nivolumab treatment and NKT cell-based immunotherapy may be a powerful tool for treatment of cancer patients.

\section{Limitations}

In this study, we examined the impact of nivolumab on anti-tumor effects of NKT cells. A limitation of this study is that the peripheral blood NKT cells were collected from healthy volunteers, not from patients with cancer. Since the number of NKT cells are decreased in cancer bearing patients, it is technically difficult to collect enough NKT cells to obtain reliable data. We thus used samples from healthy volunteers;

therefore, there is a possibility that the results might not reflect the situation of cancer patient treatment in vivo.

\section{Abbreviations}

NKT: natural killer T

NK: natural killer

TCR: T cell receptor

a-GalCer: a-galactosylceramide

PD-1: programmed death-1

PD-L: programmed death ligand

IFN-g: interferon-g

APC: antigen-presenting cell

PBMC: peripheral blood mononuclear cell

\section{Declarations}




\section{Ethics approval and consent to participate}

This study was approved by the Ethics Committee of Chiba University. Written informed consent was obtained from all participants included in this study.

\section{Consent for publication}

Not applicable.

\section{Availability of data and materials}

The datasets used and/or analyzed during the current study available from the corresponding author on reasonable request.

\section{Competing interests}

The authors declare that they have no competing interests.

\section{Funding}

This work was supported by Grants-in-Aid for Scientific Research from the Ministry of Education, Culture, Sports, Science and Technology (MEXT) (KAKENHI grant numbers $17 \mathrm{~K} 6892$ and 18H02892). The funding bodies played no role in the design of the study and data collection, analysis, interpretation of data and in writing the manuscript.

\section{Authors' contributions}

$\mathrm{NM}$ and FI contributed equally to this work. NM and FI designed and performed the experiments. FI wrote the manuscript. SM was in charge of the overall study direction and planning. All authors have read and approved the manuscript.

\section{Acknowledgements}

We thank Saori Tagi for her excellent technical assistance, and Ono Pharmaceutical Co. Ltd. for providing the nivolumab. We also thank Alison Sherwin, PhD, from Liwen Bianji, Edanz Group China (www.liwenbianji.cn/ac) for editing the English text of a draft of this manuscript.

\section{References}

1. Taniguchi M, Seino K, Nakayama $T$ : The NKT cell system: bridging innate and acquired immunity. Nat Immunol 2003, 4(12):1164-1165.

2. Rossjohn J, Pellicci DG, Patel O, Gapin L, Godfrey DI: Recognition of CD1d-restricted antigens by natural killer T cells. Nat Rev Immuno/2012, 12(12):845-857. 
3. Bedard M, Salio M, Cerundolo V: Harnessing the Power of Invariant Natural Killer T Cells in Cancer Immunotherapy. Front Immunol 2017, 8:1829.

4. Takami M, Ihara F, Motohashi S: Clinical Application of iNKT Cell-mediated Anti-tumor Activity Against Lung Cancer and Head and Neck Cancer. Front Immunol 2018, 9:2021.

5. Motohashi S, Ishikawa A, Ishikawa E, Otsuji M, lizasa T, Hanaoka H, Shimizu N, Horiguchi S, Okamoto Y, Fujii S et al: A phase I study of in vitro expanded natural killer T cells in patients with advanced and recurrent non-small cell lung cancer. Clin Cancer Res 2006, 12 (20 Pt 1):6079-6086.

6. Motohashi S, Nakayama T: Clinical applications of natural killer T cell-based immunotherapy for cancer. Cancer Sci 2008, 99(4):638-645.

7. Xu-Monette ZY, Zhang M, Li J, Young KH: PD-1/PD-L1 Blockade: Have We Found the Key to Unleash the Antitumor Immune Response? Front Immunol 2017, 8:1597.

8. Pesce S, Greppi M, Tabellini G, Rampinelli F, Parolini S, Olive D, Moretta L, Moretta A, Marcenaro E: Identification of a subset of human natural killer cells expressing high levels of programmed death 1: A phenotypic and functional characterization. J Allergy Clin Immunol 2017, 139(1):335-346 e333.

9. Vari F, Arpon D, Keane C, Hertzberg MS, Talaulikar D, Jain S, Cui Q, Han E, Tobin J, Bird R et al: Immune evasion via PD-1/PD-L1 on NK cells and monocyte/macrophages is more prominent in Hodgkin lymphoma than DLBCL. Blood 2018, 131(16):1809-1819.

10. McDermott DF, Atkins MB: PD-1 as a potential target in cancer therapy. Cancer Med 2013, 2(5):662673.

11. Topalian SL, Hodi FS, Brahmer JR, Gettinger SN, Smith DC, McDermott DF, Powderly JD, Carvajal RD, Sosman JA, Atkins MB et al: Safety, activity, and immune correlates of anti-PD-1 antibody in cancer. N Engl J Med 2012, 366(26):2443-2454.

12. Hawkes EA, Grigg A, Chong G: Programmed cell death-1 inhibition in lymphoma. Lancet Oncol 2015, 16(5):e234-245.

13. Kamata T, Suzuki A, Mise N, Ihara F, Takami M, Makita Y, Horinaka A, Harada K, Kunii N, Yoshida S et al: Blockade of programmed death-1/programmed death ligand pathway enhances the antitumor immunity of human invariant natural killer T cells. Cancer Immunol Immunother 2016, 65(12):14771489.

14. Bardhan K, Anagnostou T, Boussiotis VA: The PD1:PD-L1/2 Pathway from Discovery to Clinical Implementation. Front Immunol 2016, 7:550.

15. Carpenito C, Milone MC, Hassan R, Simonet JC, Lakhal M, Suhoski MM, Varela-Rohena A, Haines KM, Heitjan DF, Albelda SM et al: Control of large, established tumor xenografts with genetically retargeted human T cells containing CD28 and CD137 domains. Proc Natl Acad Sci U S A 2009, 106(9):3360-3365.

16. Sugamata R, Sugawara A, Nagao T, Suzuki K, Hirose T, Yamamoto K, Oshima M, Kobayashi K, Sunazuka T, Akagawa KS et al: Leucomycin A3, a 16-membered macrolide antibiotic, inhibits influenza A virus infection and disease progression. J Antibiot (Tokyo) 2014, 67(3):213-222. 
17. Filippis C, Arens K, Noubissi Nzeteu GA, Reichmann G, Waibler Z, Crauwels P, van Zandbergen G: Nivolumab Enhances In Vitro Effector Functions of PD-1(+) T-Lymphocytes and Leishmania-Infected Human Myeloid Cells in a Host Cell-Dependent Manner. Front Immunol 2017, 8:1880.

\section{Figures}

A
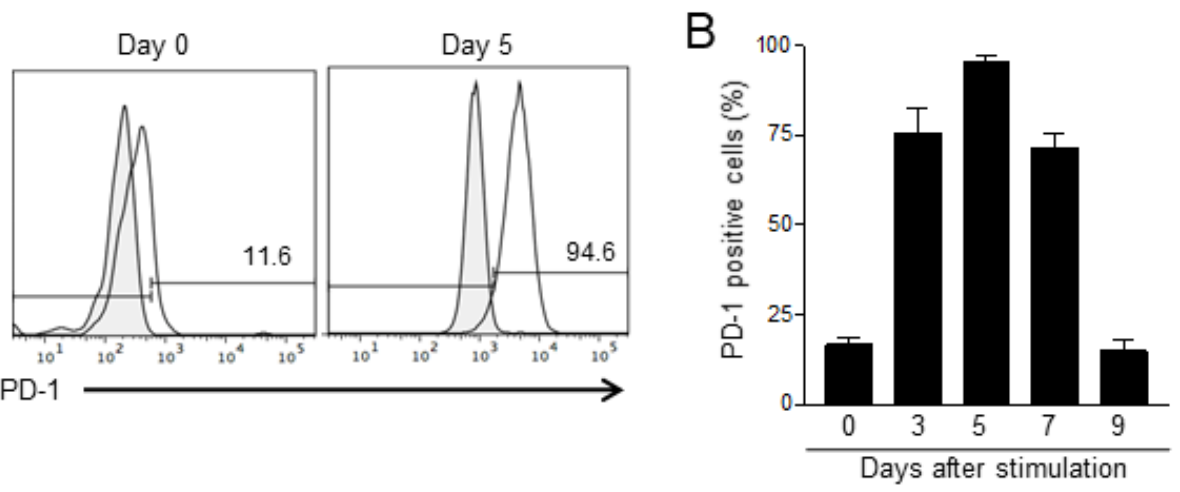

C

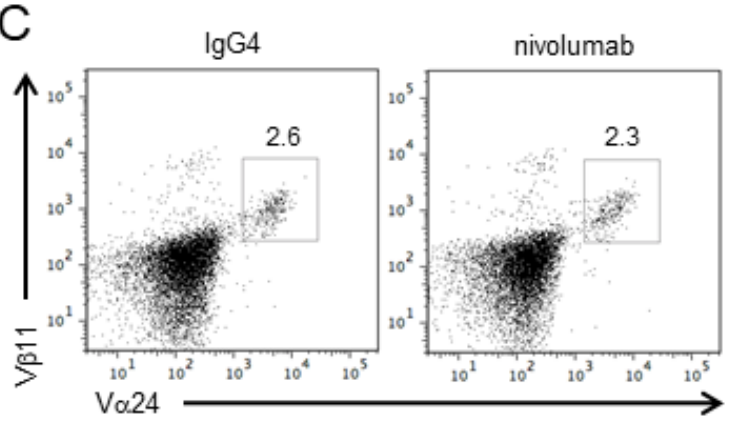

$E$
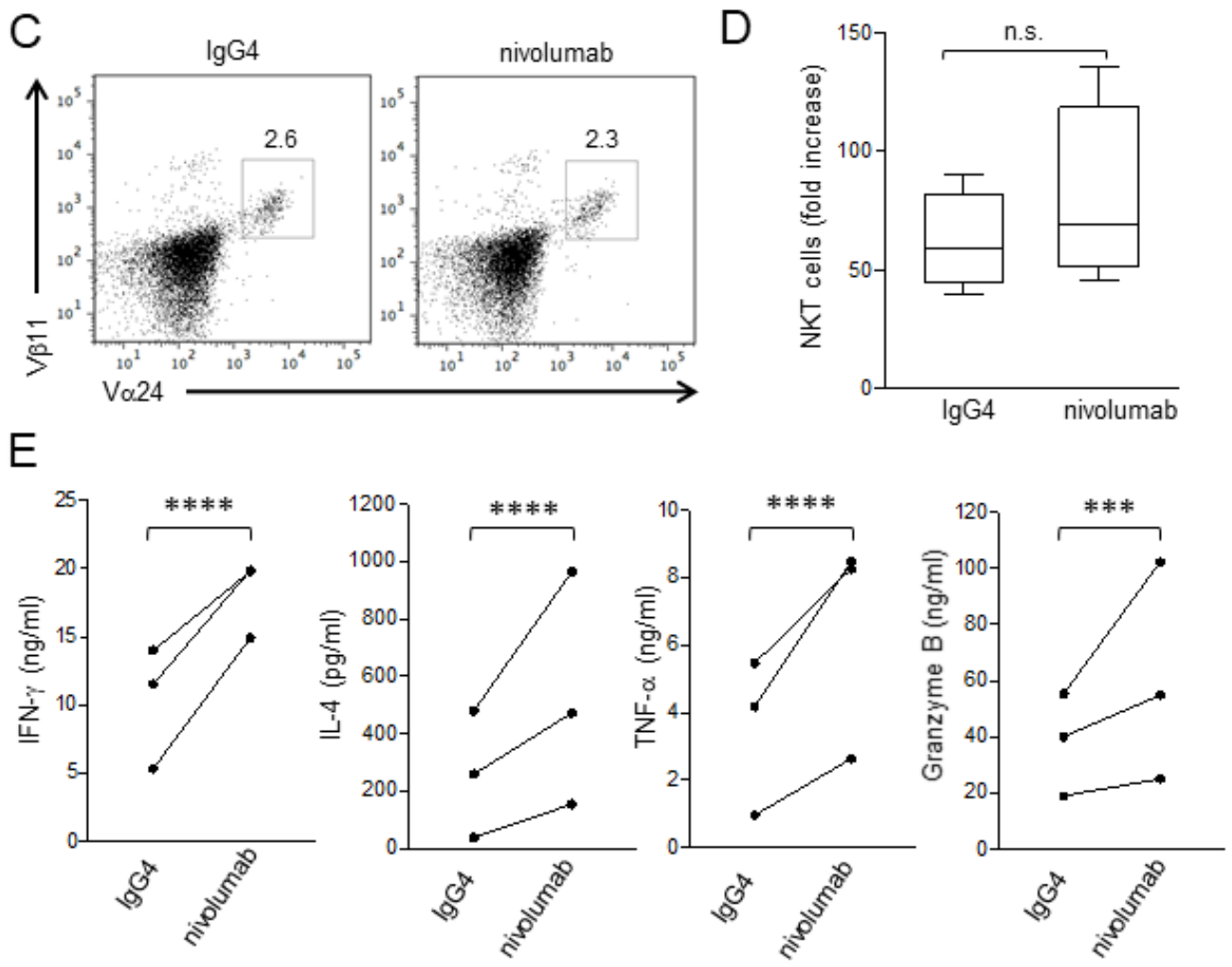

Fig. 1 
Nivolumab enhances the production of cytokines and effector molecules by NKT cells. (A, B) PD-1 expression on NKT cells. PBMCs were cultured with a-GalCer and IL-2. Cells were harvested at days 0, 3, 5, 7, and 9, stained with an anti-PD-1 antibody, and analyzed by flow cytometry. (C, D) PBMCs were cultured with a-GalCer and IL-2 in the presence of nivolumab or hlgG4 isotype control. Cells were harvested at day 6 and stained with anti-TCR Va24, anti-TCR $\beta 11$, and anti-CD3 antibodies. (D) Total numbers of live cells counted after cell harvesting on day 6 . The NKT cell number was determined by the following equation: NKT cell number $=$ total number of live cells $\times \%$ Va24+V $\beta 11+$ cells $/ 100$. (E) Purified NKT cells were stimulated with plate-bound a-GalCer-loaded CD1d/anti-CD28 antibody/PD-L1 for $24 \mathrm{~h}$, and the culture supernatants were collected. The production levels of IFN- $y$, IL-4, TNF-a, and Granzyme B were determined by cytometric bead array. P-values were calculated by a paired t-test. ${ }^{\star \star \star} P<0.001$, ${ }^{\star \star \star *} P<0.0001$, n.s.: not significant. 
A

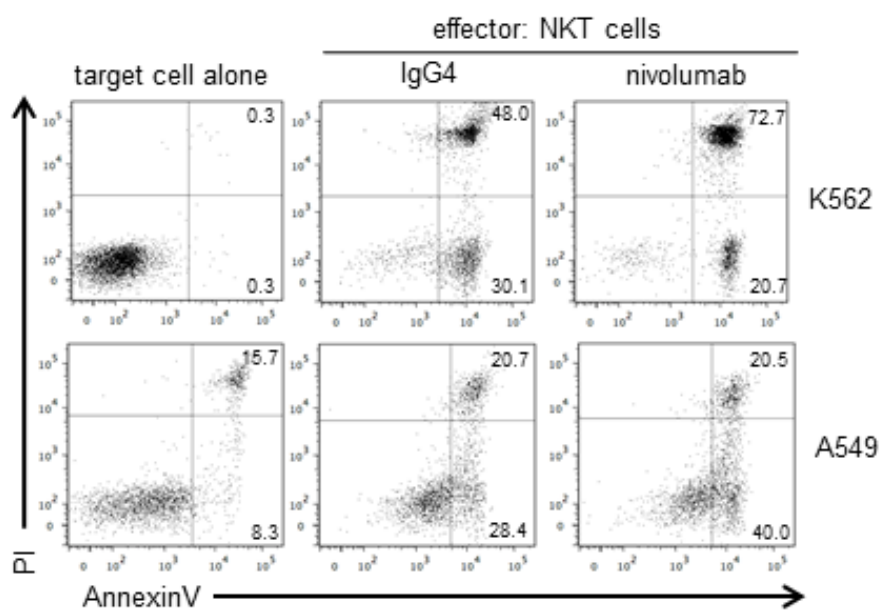

Fig. 2
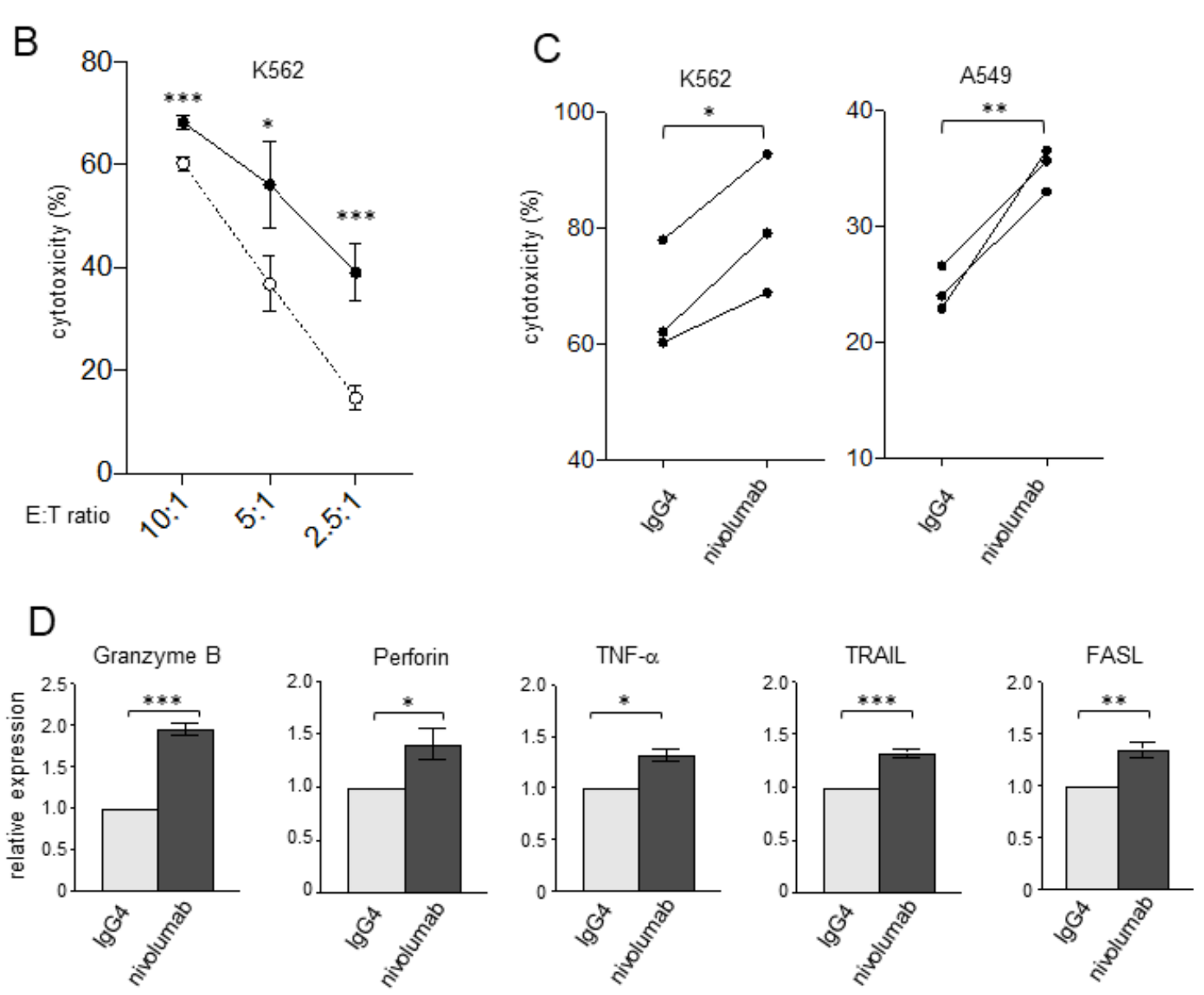

\section{Figure 2}

Nivolumab treatment enhances the tumoricidal activity of NKT cells. (A, B) Purified NKT cells treated with nivolumab or isotype hlgG4 were further treated with nivolumab or hlgG4, and then co-cultured with CTVlabeled K562 or A549 cells for $4 \mathrm{~h}$. The cells were stained with annexin V and PI, and determined for tumor cell-specific apoptosis by gating on CTV labeling. (A) Data for CTV+ K562 or A549 cells co-cultured at an E/T ratio of 10:1. (B) Cytotoxicity against K562 cells calculated for E/T ratios of 10:1, 5:1, and 2.5:1. (C) 
Purified NKT cells treated with nivolumab or isotype hlgG4 were further treated with nivolumab or hlgG4, and then co-cultured with K562 cells for $4 \mathrm{~h}$. The cells were subjected to real-time PCR to assess the expression levels of Granzyme B, Perforin, TNF-a, TRAIL, and FASL. P-values were calculated by an unpaired t-test. ${ }^{*} \mathrm{P}<0.05,{ }^{*} \mathrm{P}<0.01,{ }^{*} * \mathrm{P}<0.001$.

A

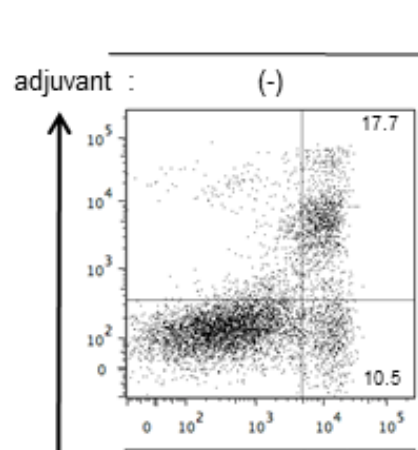

effector: NK cells

Fig.3
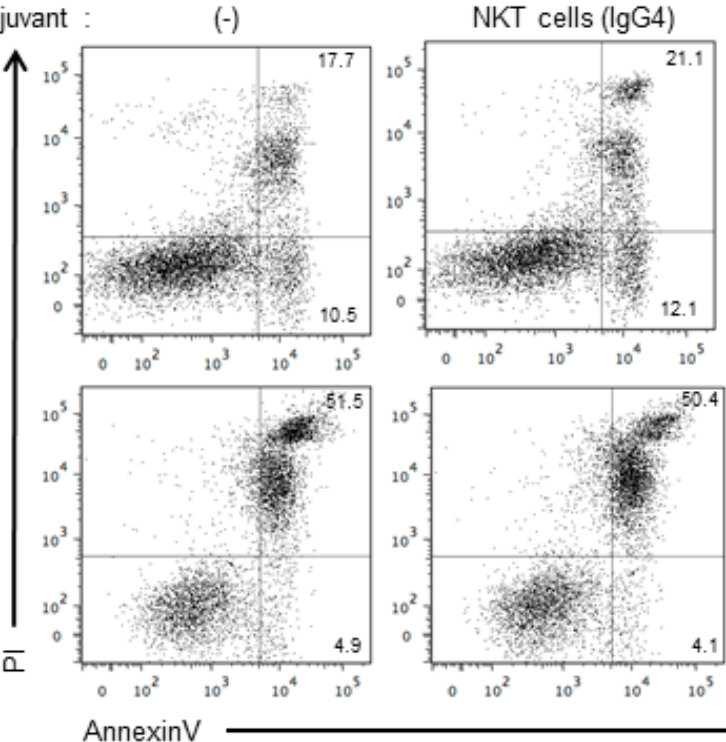
NKT cells (nivolumab)

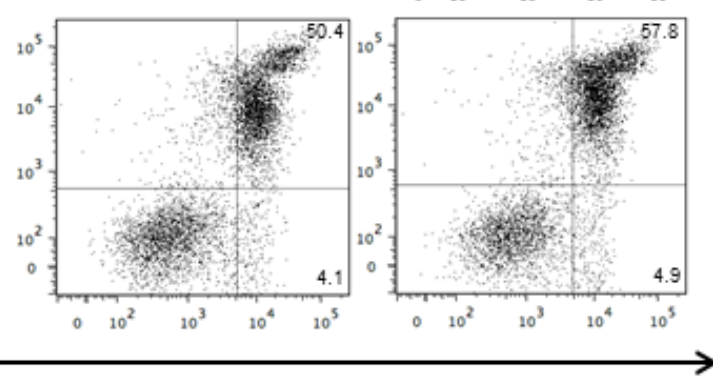

K562

A549

B
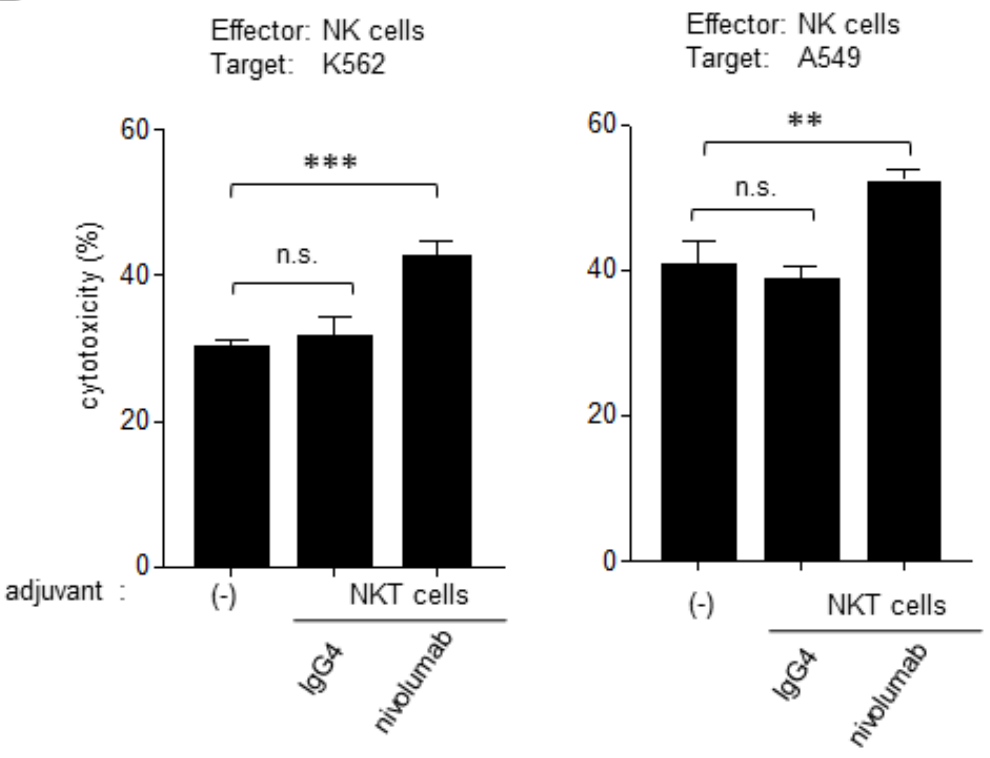

Figure 3 
Nivolumab enhances the adjuvant effect of NKT cells on NK cells. (A, B) Purified NKT cells treated with nivolumab or isotype hlgG4 were co-cultured with $\mathrm{K} 562$ cells in the top compartments of transwells, while NK cells were co-cultured with CTV-labeled K562 cells at a E/T ratio of 10:1 in the bottom compartments of the transwells. The cells in the bottom compartments were harvested after $4 \mathrm{~h}$ of culture, and stained with annexinV and PI to assess K562 cell-specific apoptosis. (A) Data for CTV+K562 cells. (B) Cytotoxicity against $K 562$ cells calculated at an E/T ratio of 10:1. P-values were calculated by an unpaired t-test. ${ }^{\star *} \mathrm{P}<0.01,{ }^{*} * \mathrm{P}<0.001$, n.s.: not significant. 